\title{
Co-channel Interference Mitigation Capability of Fixed Relays Connected by Optical Fibre
}

\author{
Rong Zhang, Xinyi Xu and Lajos Hanzo \\ School of ECS., Univ. of Southampton, SO17 1BJ, UK. \\ Tel: +44-23-80-593 125, Fax: +44-23-80-593 045 \\ Email: lh@ecs.soton.ac.uk, http://www-mobile.ecs.soton.ac.uk
}

\begin{abstract}
In this paper, we consider the co-channel interference mitigation capability of fixed relay aided wireless systems, where the relays are linked to the base station using realistic imperfect optical fibre. Three interference mitigation techniques are investigated, namely an appealing reduced-power transmission technique, the classic eigen-beamforming arrangement and their hybrid. The cellular model of Shamai and Wyner is used in conjunction with Fractional Frequency Reuse (FFR) and the tradeoffs between the cell-edge throughput and cell-centre throughput are characterised for the three interference mitigation techniques employed. Our simulation results demonstrate that the combination of the reduced-power transmission and the eigenbeamforming technique is capable of improving the achievable throughput for the MSs roaming close to the cell-edge without sacrifising the throughput of the cell-centre MSs.
\end{abstract}

\section{INTRODUCTION}

Wireless systems are capable of operating without partitioning the total bandwidth into cell-specific frequency sets, although this so-called 'unity frequency-reuse pattern' is typically achieved at the cost of an increased co-channel interference level. This interference-limited wireless access system often becomes incapable of supporting a sufficiently high throughput especially at the cell-edge. Hence, a different form of frequency reuse has been adopted [1], which rely on the so-called Fractional Frequency Reuse (FFR). According to the FFR philosophy, the users near the cell-center occupy the total bandwidth, while the cell-edge users are allowed access to only a third of the total bandwidth, in order to allow the creation of three non-interfering frequency sets. More explicitly, the frequency sets of the cell-edge users in the adjacent cells are arranged to be orthogonal in order to improve their Signal to Interference plus Noise Ratio (SINR).

Moreover, wireless relays may be employed in the cell in order to improve the achievable throughput [2], which would otherwise remain low, as a result of the pathloss between the Base Station (BS) and the cell-edge Mobile Station (MS). As a beneficial effect of the relay, an enhanced cell-edge SINR is achieved. Despite the numerous benefits of wireless relays, the capacity of a wireless relay aided system is limited by that of the 'weakest' link in the entire communication chain [3]. On the other hand, it is widely recognized that the capacity of optical fibre links is higher than that of the

Acknowledgements: The financial support of the EPSRC under the auspice of the UK-India Centre of Excellence in Wireless Communications is gratefully acknowledged. wireless medium. Therefore, the Radio over Fibre (RoF) [4] technique is eminently applicable to construct the BS to relay link and the capacity of the resultant RoF based relay system is essentially only limited by the 'last-mile' wireless link.

By using the optical fibre for the BS to relay link, the relay is typically closer to the cell-edge MS, hence providing an improved signal quality. Naturally, relaying is capable of reducing the pathloss owing to the reduced distance from the relay to the MS, but it will consequently increase the interference imposed on the adjacent cell's MSs roaming near the cell-center, which may use the same frequency as the current cell's cell-edge MS in a typical FFR arrangement. Against this background, in this paper, we investigate three different interference mitigation techniques designed for a realistically modelled optical fibre based relay aided system in the context of the Shamai-Wyner interference model [5], where we are aim for improving the attainable throughput of the cell-edge MS, while minimising the interference imposed on the adjacent cell's MS roaming near the cell-center.

The remainder of this paper is organised as follows. In Section II, we introduce the Shamai-Wyner interference model and discuss three interference mitigation techniques employed in our optical fibre based relay aided system and present our link level results for a range of modulation and coding schemes. This is followed by our performance evaluation in Section III and we conclude our discourse in Section IV.

\section{Optical Fibre Based Fixed Relay Aided System}

\section{A. Shamai-Wyner Interference Model}

Let us investigate an optical fibre based fixed relay aided system using the Shamai-Wyner interference model of [5] seen in Fig. 1, which illustrates two partially overlapping circular cells having a radius of $R$, each of which consists of three $120^{\circ}$ sectors, where the distance between the BS $O_{1}$ and BS $O_{2}$ is $\overline{O_{1} O_{2}}=\sqrt{3} R$, which is $3 \mathrm{~km}$ in urban macro scenario. We assume that the serving cell is $O_{1}$ and a cell-edge MS is placed at position 'a' of Fig. 1 with the polar coordinates denoted by $\left(\theta_{a}, L_{a}\right)=(0, \sqrt{3} R / 2)$, while the adjacent cell's near cell-center MS is placed at position 'c' of Fig. 1 having the polar coordinates of $\left(\theta_{c}, L_{c}\right)=(-\pi, R / 2)$. For the sake of improving the throughput of the cell-edge MS 'a', the relay is placed at position ' $\mathrm{g}$ ' described by the polar coordinates $(\theta, L)$. Hence, the distances from the relay ' $\mathrm{g}$ ' to both MSs may be expressed as $D_{a}=\left(L^{2}+L_{a}^{2}-2 L L_{a} \cos \theta\right)^{1 / 2}$ and $D_{c}=\left(L^{2}+L_{c}^{2}-2 L L_{c} \cos \theta\right)^{1 / 2}$, respectively. 


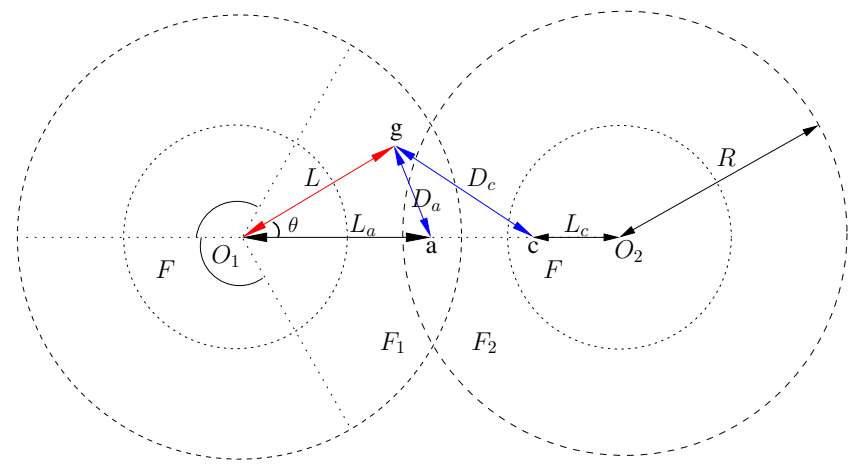

Fig. 1. Wyner interference model of two cells with radius $R$ and FFR arrangement.

Having described the associated geometric properties, let us now characterize the interference generated in this system model. Let us assume that the above-mentioned FFR regime is employed in the system, where the cell-center users of both cells have access to the entire spectral resources $F$, while the cell-edge users have access to only a third of the total bandwidth and are mutually orthogonal, hence we have $F_{a} \cap$ $F_{c}=\varnothing$. For the cell-edge MS 'a', relay aided transmission is required, where the link from $\mathrm{BS} O_{1}$ to the relay is constituted by an imperfect optical fibre, while the last-mile connection relies on wireless access. On the other hand, for the cellcenter MS 'c', a direct wireless link dispensing with relaying is established by the $\mathrm{BS} \mathrm{O}_{2}$. Hence, the transmissions from the relay to the cell-edge MS 'a' impose interference upon the cell-center MS 'c', and similarly, the transmissions from the BS $\mathrm{O}_{2}$ to its cell-center MS 'c' also inflict interference upon the cell-edge MS 'a'.

\section{B. Received Signal Strength}

We now describe the received SINR model of the link, where the BS $O_{1}$ transmits signal $x$ via the realistic optical fibre aided relay ' $g$ ' to the cell edge MS 'a'. The signal received at the relay after passing through the 'lossy' optical fibre may be written as

$$
y_{g}=A_{f}\left(A_{p} x+I_{p}\right)+n_{f} .
$$

More explicitly, we have $A_{f}=\exp (-\alpha L / 2)$ and $n_{f} \sim$ $\mathcal{C N}\left(0, \sigma_{f}\right)$, where the lossy optical fibre's attenuation coefficient is $\alpha$ and the complex-valued Additive White Gaussian Noise (AWGN) is $n_{f}$. When the wavelength of light is $\lambda=1.55 \mu \mathrm{m}$, a typical value for the attenuation coefficient is $\alpha=0.2 \mathrm{~dB} / \mathrm{km}$ [6]. Furthermore, the so-called pulsebroadening effect of the optical fibre is also taken into account, where the pulse propagated over the fibre is subject to both the peak power reduction and the Inter-Pulse-Interference (IPI). The peak power reduction is given by a factor of [7]:

$$
A_{p}=T_{0} /\left(T_{0}^{2}-i \kappa^{2} L^{2}\right)^{1 / 2},
$$

where $T_{0}=10 \mathrm{ps}$ stands for the Half-Width-Half-Maximum (HWHM) of the initial Gaussian signalling pulse, while $\kappa=$ $20 \mathrm{ps}^{2} / \mathrm{km}$ is a constant. The IPI is accumulated as a result of the pulse broadening and is a function of the optical fibre length $L$, which may be modelled as a linear function of the distance yielding $I_{p}=\gamma L$.

This noise-contaminated channel-faded signal $y_{g}$ is normalised by a power-scaling factor $\chi$ and then forwarded to the destination, namely to the cell-edge MS through the wireless channel, where the power-scaling factor $\chi$ is employed in order to maintain a constant transmit power at the relay. The signal received at the destination MS may be written as

$$
y_{a}=\psi_{g a} h_{g a} \chi y_{g}+I_{a}+n,
$$

where $\psi_{g a}, h_{g a}, I_{a}$ and $n \sim \mathcal{C N}\left(0, \sigma_{0}\right)$ represent the largescale attenuation, small-scale fast Rayleigh fading, the cochannel interference generated by cell $\mathrm{O}_{2}$ and the complexvalued AWGN, respectively. Furthermore, the subscript $(\cdot)_{i j}$ denotes the channel spanning from point ' $i$ ' to point ' $j$ '. More explicitly, we consider both the pathloss and the shadowing component, which are given by $\psi_{g a}=\left(D_{1}^{-\mu} 10^{\xi_{s} / 10}\right)^{1 / 2}$, where $\mu=4$ denotes the power-decay factor and $\xi_{s}$ is generated by zero-mean real Gaussian random variable having standard deviation of $\sigma_{s}=8 \mathrm{~dB}$.

Let $A=A_{f} A_{p}$ and $N_{f}=\left(A_{f} I_{p}\right)^{2}+2 \sigma_{f}^{2}$ denote the equivalent optical fibre attenuation factor and equivalent optical fibre noise variance, the SINR of MS 'a' in cell $O_{1}$ is given by:

$$
\gamma_{a}=\frac{\left(\psi_{g a}\left|h_{g a}\right| \chi A\right)^{2}}{2 \sigma_{0}^{2}+\left(\psi_{g a}\left|h_{g a}\right| \chi\right)^{2} N_{f}+\left(\psi_{O_{2} a}\left|h_{O_{2} a}\right|\right)^{2}},
$$

where the second term and the third term in the denominator represent the noise generated by the components in the lossy optical fibre and the co-channel interference generated by the adjacent cell's transmission. On the other hand, MS 'c' near the cell-center of $\mathrm{O}_{2}$ receives both the signal transmitted from $\mathrm{O}_{2}$ via the wireless channel and the interference imposed by the relay in cell $O_{1}$. Hence, the SINR at the input of the receiver of MS 'c' in cell $\mathrm{O}_{2}$ is given by:

$$
\gamma_{c}=\frac{\left(\psi_{\mathrm{O}_{2}}\left|h_{\mathrm{O}_{2} c}\right|\right)^{2}}{2 \sigma_{0}^{2}+\left(\psi_{g a}\left|h_{g a}\right| \chi\right)^{2} N_{f}+\left(\psi_{g c}\left|h_{g c}\right| \chi A\right)^{2}},
$$

where the second term and the third term in the denominator represent the noise generated by the components in the lossy optical fibre and the co-channel interference generated by the transmissions of the relay.

Fig 2 shows the average SINR of both MS 'a' and of MS 'c' as a function of the relay position ' $g$ ' described by the distance ratio of $\rho=L / R$ and angular rotation of $\theta=0$ and IPI of the optical fibre is ranging from $\gamma=0.2,0.5,0.7$, where the average was calculated for 1000 simulation runs when assuming that the normalised optical fibre link SNR is $10 \mathrm{~dB}$ and the normalised wireless link SNR is $2 \mathrm{~dB}$. It can be seen that all SINR curves recorded for MS 'a' are monotonically increasing and saturate at high values of $\rho$, while all SINR curves plotted for MS 'c' are monotonically decreasing. These two distinct trends indicate the complementary characteristics experienced by the two MSs, namely that increasing the SINR for one of the MSs will inevitably impose an SINR degradation on the other MS. When the accumulated IPI is increased, the SINR of cell-edge MS 'a' decreases dramatically while that of the cell-center MS 'c' is slightly reduced. 


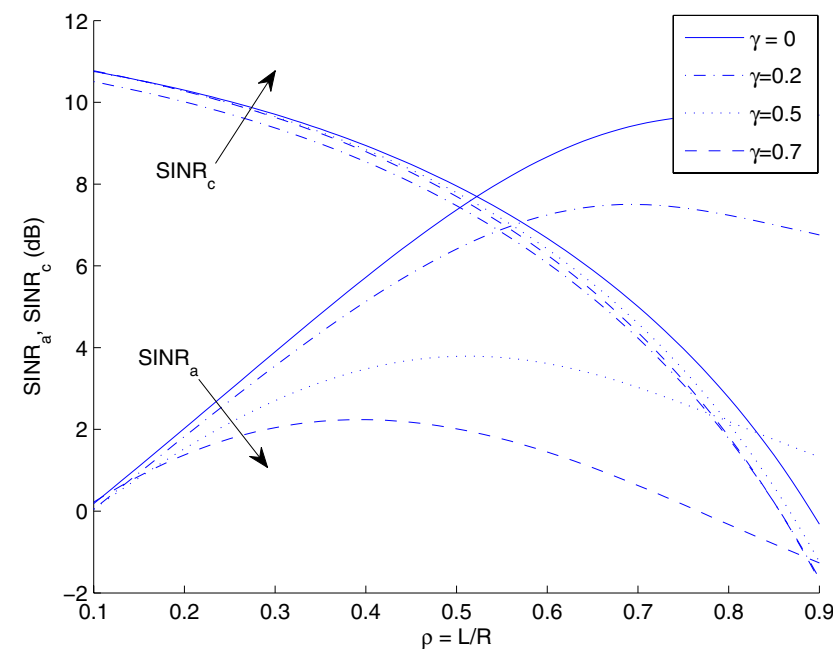

Fig. 2. SINR of both the cell-edge MS 'a' and the cell-center MS 'c' having different amount of IPI.

\section{Interference Mitigation Techniques}

Intuitively, placing a relay close to the cell-edge will improve its reception quality, while imposing increased interference upon the neighbouring cell. Hence the natural design methodology of a relay aided system is to improve the throughput of the cell-edge MSs, while minimising the throughput reduction inflicted upon the neighbouring cell owing to the increased interference. We thus discuss three different interference mitigation techniques, namely a beneficial reduced-power transmission technique, the classic eigenbeamforming technique and a hybrid combination of them.

1) Reduced-power technique: This 'green' reduced-power technique refers to the arrangement, where the transmit power of the relay is scaled down by the normalisation factor of $\chi=\beta /\left(A^{2}+N_{f}\right)^{1 / 2}$ with $\beta \in(0,1]$. The underlying principle of this technique is that the interference imposed on MS 'c' may be substantially reduced by the reduction of the relay's the transmission power. This technique becomes identical to the classic direct-relaying, when we have $\beta=1$.

2) Eigen-beamforming technique: In this paper, we stipulate the simplifying assumption that the instantaneous CSI is perfectly known at the relay's transmitter, which is a uniformly spaced linear array of four antenna elements while the two MSs are equipped with a single receive antenna. The wellknown eigen-beamforming technique [8] is considered, where the transmit antenna weights are given by the eigenvector $\mathbf{v} \in \mathcal{C}^{4 \times 1}$ corresponding to the largest eigenvalue of the spatial channel's covariance matrix in the Multiple Input Single Output (MISO) system, namely $\mathbf{H}_{\text {ga }} \in \mathcal{C}^{1 \times 4}$. As a result, the equivalent fast fading channels become $h_{g a}=\mathbf{H}_{\mathbf{g a}} \mathbf{v}$ and $h_{g c}=\mathbf{H}_{\mathrm{gc}} \mathbf{v}$, respectively and hence the channel's amplitude $\left|h_{g a}\right|$ is maximised, while $\left|h_{g c}\right|$ remains the same. The underlying principle of this technique is that by transmitting in the direction associated with the maximum equivalent channel gain of $\left|h_{g a}\right|$, the received SINR of the cell-edge MS 'a' is improved at a given transmit power, while the co-channel interference imposed on MS ' $c$ ' remains unchanged.

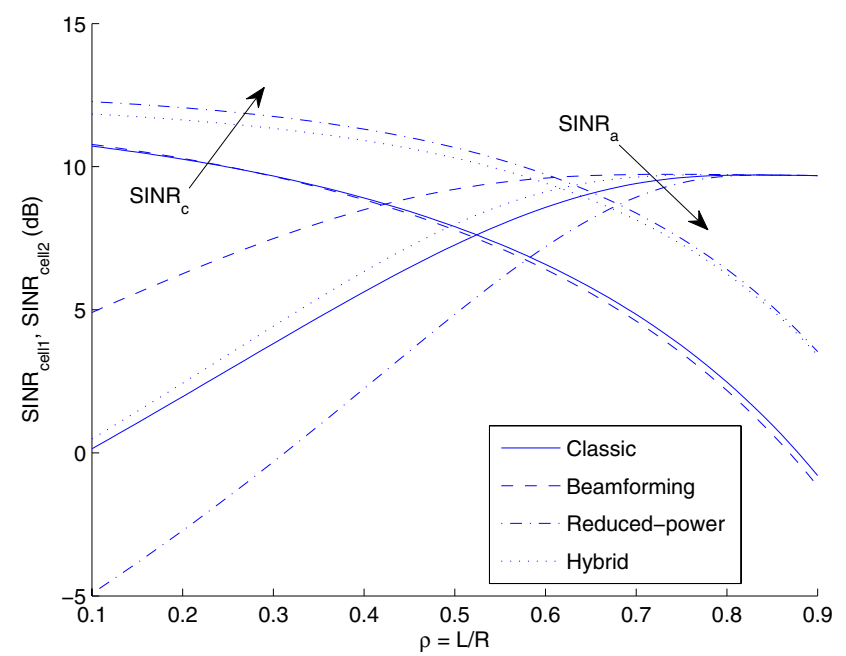

Fig. 3. SINR of both the cell-edge MS ' $a$ ' and the cell-center MS ' $c$ ' employing different interference mitigation techniques and assuming no IPI of the optical fibre, i.e. $\gamma=0$.

3) Hybrid technique: We may combine the benefits of the above two techniques, leading to a hybrid technique. Specifically, the co-channel interference imposed on MS ' $c$ ' may be mitigated by the reduced-power solution, while the resultant degraded SINR at the cell-edge MS 'a' imposed by the reduced-power transmission may be compensated by the eigen-beamforming gain of our directional technique, because it enhances the SINR of the cell-edge MS 'a', while at the same time maintaining a reduced co-channel interference.

Fig 3 shows the average SINR of both MS 'a' and of MS ' $c$ ' as a function of the position of relay ' $g$ ' described by the distance ratio of $\rho=L / R$ and angular rotation of $\theta=0$ and assuming no IPI of the optical fibre, i.e. $\gamma=0$, where the average was calculated for 1000 simulation runs when assuming that the normalised optical fibre link SNR is 10 $\mathrm{dB}$ and the normalised wireless link SNR is $2 \mathrm{~dB}$. When comparing the three interference mitigation techniques with respect to the classic benchmarker curves represented by the solid lines of Fig 3, it is clearly seen that employing the eigenbeamforming technique represented by the dash lines of Fig 3 improves the SINR of the cell-edge MS 'a', while maintaining the SINR of the cell-center MS 'c'. By contrast, employing the reduced-power technique represented by the dash-dot lines of Fig 3, when transmitting at a quarter of the full power, namely using $\beta=1 / 2$, substantially improves the SINR of the cellcenter MS 'c' by sacrificing the SINR of the cell-edge MS 'a'. By amalgamating the benefits of both techniques, the hybrid solution represented by dotted lines of Fig 3 leads to both a beneficial SINR improvement for MS 'c' and to a noticeable SINR improvement for the cell-edge MS 'a'.

\section{System Throughput}

Although different interference mitigation techniques result in different SINR characteristics with respect to the relay's position, the ultimate system performance metric is the achievable throughput. This is because an increased SINR 
does not necessarily lead to an increased throughput in the absence of adaptive transceivers, hence transmitting at a power higher than absolutely necessary for achieving the required throughput is irrational. The throughput of the system may be defined as the maximum successfully transmitted information rate, which is referred to as the system's goodput, given by:

$$
\eta(\gamma)=R_{M} R_{C}\left[1-P_{b l}^{b}(\gamma)\right]
$$

where $R_{M}$ and $R_{C}$ denote the rate of the modulation scheme and channel code, respectively, while $P_{b l}^{b}$ represents the BLock Error Rate (BLER) corresponding to the particular Modulation and Coding Scheme (MCS) employed. In this paper, we assume that Bit Interleaved Coded Modulation (BICM) [9] is employed, which relies on the M-ary Quadrature Amplitude Modulation (QAM) [10], where we have $M=2^{b}$ and $b=$ $2,4,6$ represents the number of bits per QAM symbol, namely the rate $R_{M}=b$.

Let us now highlight the derivation of the BLER $P_{b l}^{b}$ of a BICM scheme based on the BLER $P_{b l}^{0}$ of a binary channel code as a function of the received SINR $\gamma$ in AWGN channel. The BLER $P_{b l}^{0}$ is a monotonic function of the input SINR experienced by the particular binary channel code employed, which may be referred to as the equivalent SINR $\gamma_{0}$. The equivalent SINR $\gamma_{0}$ is also a monotonic function of the mutual information per binary bit $I_{0}$. When M-ary QAM is considered, $I_{0}$ may be expressed as in Eq (7) [9], where $w \sim \mathcal{C N}(0,1 / 2 \gamma)$ is the zero-mean complex Gaussian noise and $u \in \mathcal{A}$ represents the constellation points in the QAM alphabet $\mathcal{A}$. For $b=1$, the equivalent SINR $\gamma_{0}$ may be expressed as $\gamma_{0}=I_{0}^{-1}\left[1, I_{0}(b, \gamma)\right]$. Hence, the final BLER of a BICM scheme $P_{b l}^{b}$ may be written as:

$$
P_{b l}^{b}(\gamma)=P_{b l}^{0}\left\{I_{0}^{-1}\left[1, I_{0}(b, \gamma)\right]\right\},
$$

Again, we employ M-ary QAM modulation combined with Rate Compatible Punctured Turbo Codes (RCPTC) [11] having six selected MCSs, namely Mode $1, \ldots, 6$ of $\left[R_{M}, R_{C}\right]=$ $[(2,1 / 2),(2,3 / 4),(4,1 / 2),(4,3 / 4),(6,2 / 3),(6,6 / 7)]$. Then the associated throughput in terms of 'bits/s/Hz' may be obtained by assuming a Nyquist roll-off factor of zero and $P_{b l}^{0}$ is obtained by Monte Carlo simulation of the RCPTC of rate $R_{C}=[1 / 2,3 / 4,2 / 3,6 / 7]$ employing BPSK modulation in AWGN channel. Due to space limitation, the detailed linklevel simulation results are not shown here.

\section{Performance Evaluation}

The throughput calculation introduced in Section II-D facilitates the analysis of the throughput of different interference mitigation schemes as a function of the relay position ratio $\rho$ and $\theta=0$. We firstly assume that no IPI is observed, namely we have $\gamma=0$ and we further assume that the normalised optical fibre link SNR is $10 \mathrm{~dB}$ and the normalised wireless link SNR is $2 \mathrm{~dB}$. The corresponding throughput tradeoff is illustrated in Fig 4, where the SINR $\gamma$ averaged over 1000 simulation runs was substituted into Eq (8) and Eq (6).

\section{A. Throughput of various interference mitigation techniques}

The corresponding throughput tradeoff is observed for all plots of Fig 4, where increasing the throughput of one of the
MSs results in the throughput reduction of the other. For the classic scheme, the throughput of MS 'c' drops from $\eta=2.7$ bits/s/Hz to $\eta=0$ bits $/ \mathrm{s} / \mathrm{Hz}$, as the ratio $\rho$ increases along the x-axis, while the throughput of MS 'a' increases from $\eta=0$ bits $/ \mathrm{s} / \mathrm{Hz}$ to $\eta=2 \mathrm{bits} / \mathrm{s} / \mathrm{Hz}$, attaining the maximum throughput at the position ratio of $\rho=0.5$.

1) Eigen-beamforming technique: Since the eigenbeamforming technique is capable of increasing the SINR at MS 'a', when compared to the classic technique as seen in Fig 3, we may observe a throughput improvement when the position ratio $\rho$ is low and hence the maximum throughput of $\eta=2 \mathrm{bits} / \mathrm{s} / \mathrm{Hz}$ is achieved, when the relay is positioned at the position ratio of $\rho=0.27$ as seen in the second plot of Fig 4. However, since the total transmission power remains unchanged for this technique, the co-channel interference remains the same as for the classic technique and the throughput of MS 'c' also remains the same.

2) Reduced-power technique: When comparing the reduced-power technique to the classic technique, we may expect a right shift of the throughput curves of both MS 'a' and of MS 'c', simply because the transmission power is reduced to one fourth of the full power, namely we have $\beta=1 / 2$. It can be seen in the third plot of Fig 4 that the maximum achievable throughput of the cell-center MS ' $c$ ' becomes $\eta=3 \mathrm{bits} / \mathrm{s} / \mathrm{Hz}$ and is kept constant, when the position ratio is in the range of $\rho \leq 0.4$. The throughput is then gradually reduced to $\eta=1 \mathrm{bits} / \mathrm{s} / \mathrm{Hz}$, when $\rho$ is increased. This substantial improvement of the cell-center throughput compared to the classic technique is achieved at the cost of a reduced cell-edge throughput for MS 'a', which attains a maximum of $\eta=2$ bits $/ \mathrm{s} / \mathrm{Hz}$ at $\rho=0.6$.

3) Hybrid technique: When combining the benefits of the above two techniques, the throughput of the cell-center MS 'c' is substantially increased, similarly to the reduced-power technique, while the throughput of the cell-edge MS 'a' is maintained, as in the case of the classic technique. This is because the throughput reduction of the cell-edge MS 'a' imposed by the reduced-power technique is compensated by the eigen-beamforming gain, which does not impose additional co-channel interference. Hence the hybrid technique is capable of improving the throughput of the cell-edge MS upto its maximum of $\eta=2 \mathrm{bits} / \mathrm{s} / \mathrm{Hz}$, while at the same time maintaining the maximum attainable throughput of the cellcenter MS of $\eta \approx 3$ bits/s/Hz in the fourth plot of Fig 4 at the position ratio of $\rho=0.45$. This justifies the superiority of the hybrid technique compared to the other techniques.

\section{B. Effects of IPI}

When the IPI is taken into account and we have $\gamma=0.2$, the throughput of cell-edge MS 'a' is shown in the first plot of Fig 5, which obeys similar trends to the no IPI scenario shown in the fourth plot of Fig 4. However, when $\gamma=0.5$ is considered, as shown in the second plot of Fig 5, the celledge MS 'a' employing the hybrid technique suffers from a dramatic throughput reduction compared to the fourth plot of Fig 4. The reduction of the cell-edge throughput is imposed by the SINR reduction, when IPI is encountered as shown in 

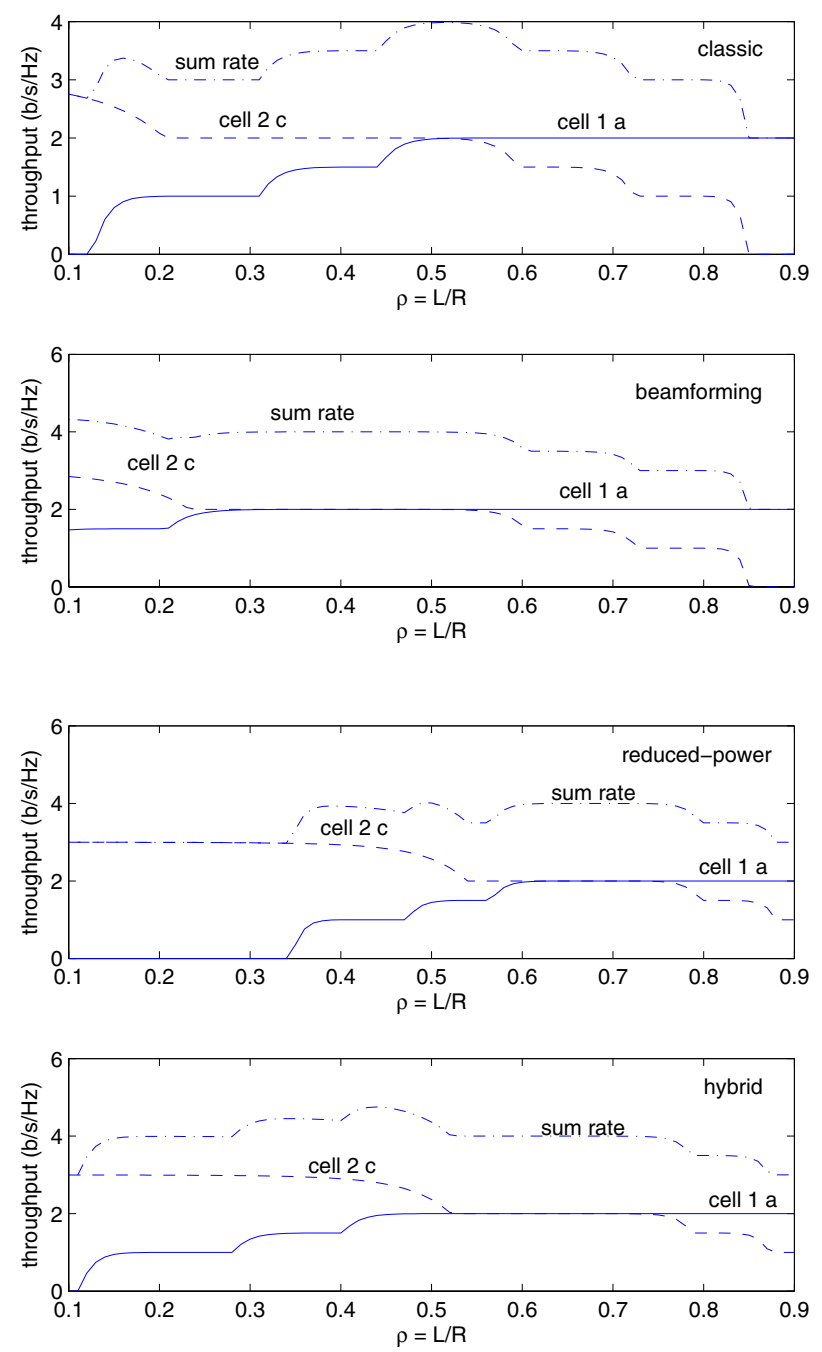

Fig. 4. Throughput of different interference mitigation techniques compared to the class technique as a function of the position ratio $\rho$ and assuming no IPI of the optical fibre, i.e. $\gamma=0$.

Fig 2. Hence, we may conclude that as long as the IPI is not severe, e.g. $\gamma<0.2$, the hybrid technique remains effective.

\section{CONCLUSions}

In this paper, we considered the SINR as well as throughput tradeoffs between supporting a cell-center MS and a celledge MS using a lossy optical fibre based fixed relay aided and FFR assisted interference limited wireless system, where the Shamai-Wyner cellular model was employed and three interference mitigation techniques were investigated. Our simulation results demonstrated that the reduced-power technique and the eigen-beamforming arrangement are only capable of improving either the throughput of the cell-center MS or
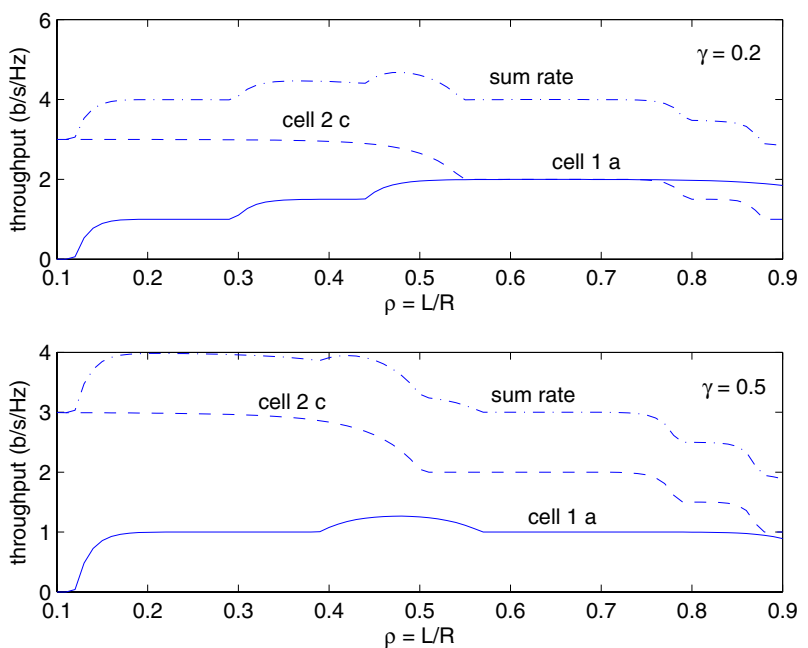

Fig. 5. Throughput of the hybrid technique as a function of the position ratio $\rho$ and assuming the presence of IPI of the optical fibre, i.e. $\gamma=0.2,0.5$.

of the cell-edge MS, while the hybrid technique is capable of maximising the throughout of the cell-edge MS without imposing any throughput reduction on the cell-center MS.

\section{REFERENCES}

[1] A. Ghosh, D. Wolter, J. Andrews, and R. Chen, "Broadband wireless access with WiMax/802.16: current performance benchmarks and future potential," IEEE Communications Magazine, vol. 43, pp. 129-136, Feb. 2005.

[2] R. Pabst, B. Walke, D. Schultz, and et al, "Relay-based deployment concepts for wireless and mobile broadband radio," IEEE Communications Magazine, vol. 42, pp. 80-89, Sept. 2004.

[3] T. Cover and J. Thomas, Elements of Information Theory. New York: Wiley, 1991.

[4] D. Wake, M. Webster, G. Wimpenny, and et al, "Radio over fiber for mobile communications," in IEEE International Topical Meeting on Microwave Photonic, Oct. 2004, pp. 157-160.

[5] S. Shamai and A. Wyner, "Information-theoretic considerations for symmetric, cellular, multiple-access fading channels," IEEE Transactions on Information Theory, vol. 6, pp. 1877-1894, Nov. 1997.

[6] G. P. Agrawal, Fiber Opticl Communications Systems, Third, Ed. John Wiley \& Sons, Inc., 2002.

[7] J. Shaw, Mathematical principles of optical fiber communications. Philadelphia: Society for Industrial and Applied Mathematics, 2004

[8] D. Tse and P. Viswanath, Fundamentals of Wireless Communication. Cambridge University Press, 2005.

[9] G. Caire, G. Taricco, and E. Biglieri, "Bit-interleaved coded modulation," IEEE Transactions on Information Theory, vol. 44, pp. 927-946, May 1998.

[10] L. Hanzo, S. X. Ng, T. Keller, and W. T. Webb, Quadrature Amplitude Modulation: From Basics to Adaptive Trellis-Coded, TurboEqualised and Space-Time Coded OFDM, CDMA and MC-CDMA Systems. Wiley-IEEE Press, 2004.

[11] J. Hagenauer, "Rate-compatible punctured convolutional codes (RCPC codes) and their applications," IEEE Transactions on Communications, vol. 36, pp. 389-400, Apr. 1988.

$$
I_{0}(b, \gamma)=1-\frac{2^{-b}}{b} \sum_{i=1}^{b} \mathbb{E}_{w}\left\{\ln \left[1+\sum_{j=1, j \neq i}^{b} \exp \left(-\frac{\left|u_{j}-u_{i}+w\right|^{2}-|w|^{2}}{1 / \gamma}\right)\right]\right\}
$$

